\title{
ARTICLE
}

\section{Measurement of depth-directional radioactive cesium distribution in soil with several detectors}

\author{
Naoki Zushi, Yoshihisa Ono, Fuminobu Sato, Yushi Kato and Toshiyuki Iida* \\ Graduate School of Engineering, Osaka University, 2-1 Yamada-oka, Suita-shi, Osaka, 565-0871, Japan
}

\begin{abstract}
It was found that the depth-directional distribution of radioactive cesium in soil could be roughly estimated with imaging plate strips, glass dosimeter fibers or a tube with built-in Si PIN photodiodes, which were put into the ground. Especially, it was confirmed that ordinary and inexpensive Si PIN photodiodes could be satisfactorily used as beta-ray detectors. The response of the photodiode to beta-rays and gamma-rays was examined by use of a ${ }^{137} \mathrm{Cs}$ standard checking source. The pulse height distributions of the photodiode showed that it had sufficiently higher detection efficiency for beta-rays than for gamma-rays and also detection signals of beta-rays from radioactive cesium could be successfully discriminated from gamma-ray background and electromagnetic noises.
\end{abstract}

Keywords: radioactive cesium contamination; radioactive cesium distribution; Si PIN photodiode; beta-ray detection

\section{Introduction}

A large quantities and variety of radionuclides were released from Fukushima Dai-ichi Nuclear Power Plant damaged by the catastrophic earthquake and tsunami on 11 March, 2011. At present (one year and a half after the disaster), ${ }^{134} \mathrm{Cs}$ and ${ }^{137} \mathrm{Cs}$ are major radionuclides in soil owing to their long half-life and the large amount of emission. Measurements of these residual radionuclides in soil are required over wide areas.

It is well known that the redistribution of deposited radionuclides has been formed in depth direction because of the infiltration of precipitation and others. Data on the depth-directional distribution of fallout radionuclides including radioactive cesium were reported for several areas after nuclear weapon tests, the Chernobyl nuclear power plant accident $[1,2]$ and the NPP accident in Fukushima[3]. It was found that most deposited radionuclides remained in depth of several centimeters but their distribution varied with soil composition.

The authors have reported a simple method of measuring depth-directional distribution of radioactive cesium in soil by use of imaging plates (IPs) [4] or radiophotoluminescence (RPL) glass dosimeters [5]. Strips of IPs or RPL glass fibers were put into the ground. By both methods radioactive cesium in soil can be detected simply, although they need enough exposure time for the IP strips and RPL glass fibers and moreover the dose reading process after the exposure to radiations

*Corresponding author. Email: iida@eie.eng.osaka-u.ac.jp in soil.

In this study, a prompt beta-ray detection system was developed by use of Si PIN photodiodes for the monitoring of radioactive cesium contamination in soil. The photodiodes followed by compact electronic circuits were put into the ground. Pulse signals due to the detection of beta-rays from radioactive cesium were counted in real time.

\section{Materials and methods}

\subsection{Selection of photodiodes}

In this study, it is first important to select a type of photodiode suitable for the detection of beta-rays from radioactive cesium in soil. From the point of the spatial resolution, the beta-ray detection has an advantage over the gamma-ray detection because beta-rays have much shorter range in soil than gamma-rays, and count data on the beta-ray detection give more accurate information on the concentration of radioactive cesium near a detector. For this purpose, therefore, a photodiode necessarily has its depletion layer (radiation-sensitive layer) of proper thickness to detect beta-rays. Too thick depletion layer increases sensitivity only for gamma-rays, which results in the degradation of the spatial resolution. As described later (see Figure 3, Tables 1 and 2), it may be a conclusion that an inexpensive and ordinary infrared photodiode, which is often used as a sensor for a general remote-controlled device, is suitable for the detection of beta-rays with low gamma-ray sensitivity. From the comparison of beta-ray detection characteristics among 
commercially available and typical photodiodes, Si PIN photodiodes (BPW34F, OSRAM) were selected as beta-ray detectors usable in soil. According to a data sheet on the photodiode (BPW34F), its photosensitive area is $2.6 \mathrm{~mm} \times 2.6 \mathrm{~mm} \times 0.05 \mathrm{~mm}$ and was covered with thin Ti foil for actual use in soil.

\subsection{Construction of T-shaped tube with built-in Si PIN photodiodes}

Figure 1 shows a schematic drawing of the detector of radioactive cesium in soil. Four pairs of the Si PIN photodiodes $(\mathrm{A} \sim \mathrm{D})$ were set in a T-shaped tube, which was put into the ground. One of the pair responds to beta-rays and background gamma-rays and the other to only background gamma-rays. Net counts of beta-rays were obtained from the difference in counts from the photodiode pair. Radioactivity on and under the ground was measured with the photodiode pairs $(\mathrm{A}, \mathrm{B})$ and $(\mathrm{C}$, D), respectively.

There was a window in the position of each photodiode pair so that it could detect beta-rays from soil fronting on the window. Each window was covered with thin Ti film for the reduction of electromagnetic noises and for the protection of the photodiode against moisture in soil.

Pulse signals from each photodiode were amplified, shaped and fed to a counter through a pulse height discriminator. Simple electronic circuits were developed and were set in a compact box with a display, and data on change in pulse counts were recorded with a personal computer (PC). A multichannel pulse height analyzer (MCA) was also used for the examination of the basic characteristics of the photodiodes.

\section{Experiments}

Basic characteristics of the Si PIN photodiode (BPW34F) were examined by use of a ${ }^{137} \mathrm{Cs}$ standard beta-ray source $(5.2 \mathrm{kBq})$. Pulse height distributions of the photodiode were measured for beta-rays and gamma-rays from the ${ }^{137} \mathrm{Cs}$ standard source. Figure 2 shows a schematic drawing of the calibration setup of the tube with built-in the photodiode. Stainless steel plates of $2 \mathrm{~mm}$ thick with a hole of a different size were used for the adjustment of beta-ray intensity on the photodiode. Beta-rays from the source are also narrow collimated by the hole of the tube. The beam intensity was determined from the comparison of the IP image of the beam spot with that of the ${ }^{137}$ Cs standard source. A 2 mm thick stainless steel plate without a hole perfectly shielded the photodiode from ${ }^{137} \mathrm{Cs}$ beta-rays. The ${ }^{137} \mathrm{Cs}$ gamma-ray flux at the position of the photodiode was estimated by a solid angle and a distance between the standard source and the photodiode.

Sample soil including radioactive cesium was prepared to test the T-shaped tube with built-in the photodiodes. The construction of the sample soil is shown in Figure 1. The sample soil was made up of two soil layers. The upper layer was made of soil in
Fukushima and the lower in Osaka. The specific radioactivity $\left({ }^{134} \mathrm{Cs}\right.$ and $\left.{ }^{137} \mathrm{Cs}\right)$ of the upper layer was about $3 \times 10^{2} \mathrm{~Bq} / \mathrm{g}$ and that of the lower layer was about $5 \mathrm{~Bq} / \mathrm{kg}$. Also, the specific radioactivity $\left({ }^{40} \mathrm{~K}\right)$ of the upper and lower layers was about $650 \mathrm{~Bq} / \mathrm{kg}$ and 480 $\mathrm{Bq} / \mathrm{kg}$, respectively. The radioactivity of these samples was measured with a Ge detector. The T-shaped tube was put into the sample soil, and pulse signals from the four photodiode pairs were counted through pulse height discriminators.

\section{Results and discussions}

\subsection{Pulse height distributions of Si PIN photodiode for ${ }^{137}$ Cs radiation source}

Figure 3 shows typical examples of pulse height distributions of the Si PIN photodiode (BPW34F) for the ${ }^{137} \mathrm{Cs}$ standard radiation source. A dark line corresponds to the response of the photodiode irradiated with radiations from the ${ }^{137} \mathrm{Cs}$ standard source as shown in Figure 2. This pulse height distribution clearly has a broad peak around channel 350 on a continuous distribution. This peak is considered to have been formed by internal conversion electrons due to the isomeric transition of ${ }^{137 \mathrm{~m}} \mathrm{Ba}$.

A light line corresponds to the response of the photodiode to ${ }^{137} \mathrm{Cs}$ gamma-rays because of the stainless steel plate without a hole. It is clear from the comparison

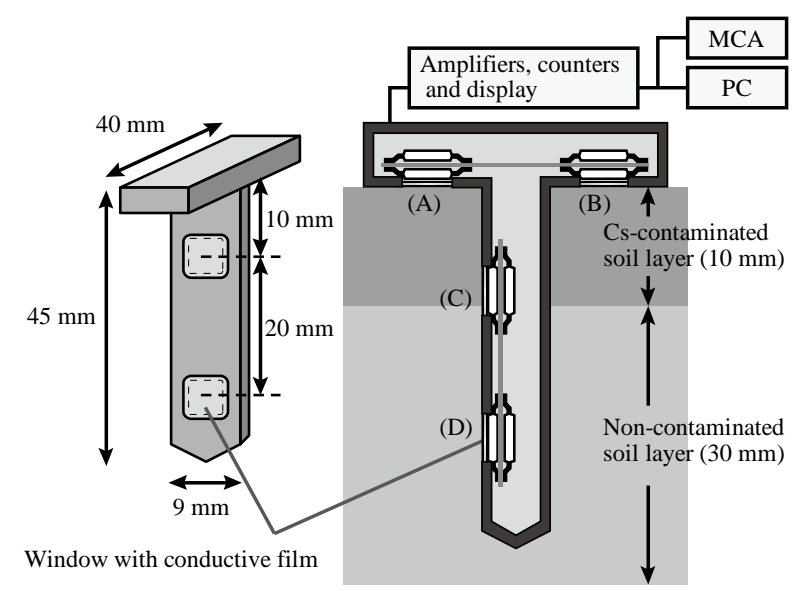

Fig. 1. Schematic drawing of T-shaped tube with built-in photodiodes for monitoring radioactive cesium in soil and construction of sample soil.

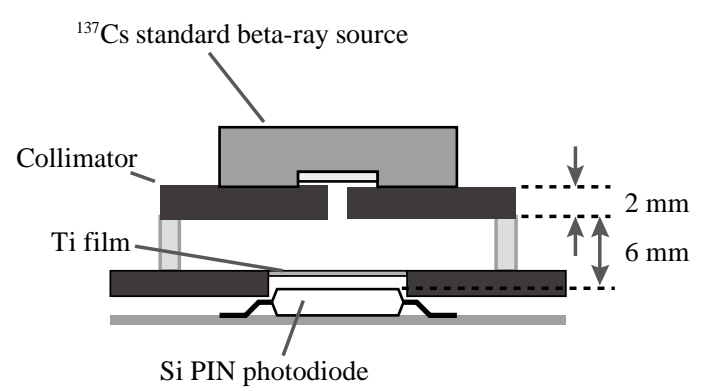

Fig. 2. Schematic representation of experimental arrangement with ${ }^{137} \mathrm{Cs}$ standard beta-ray source. 


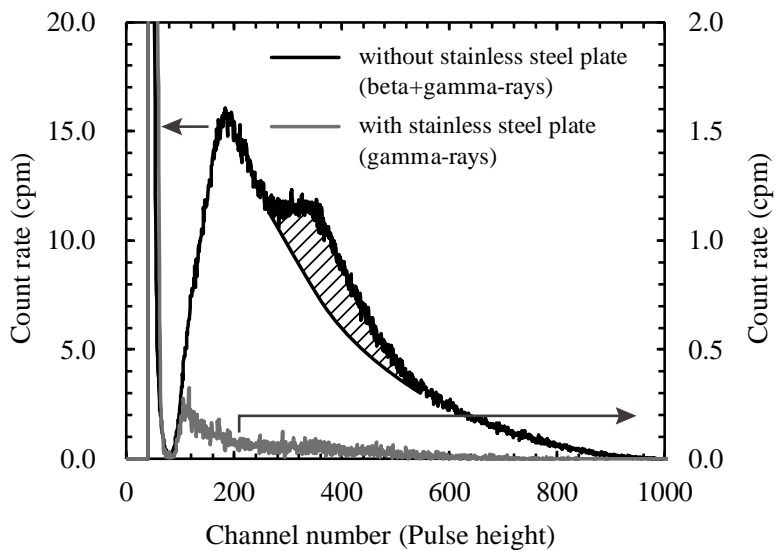

Fig. 3. Pulse height distributions of Si PIN photodiode (BPW34F) for ${ }^{137} \mathrm{Cs}$ standard radiation source.

between the dark and light lines in Figure 3 that the photodiode has sufficiently higher detection efficiency for ${ }^{137} \mathrm{Cs}$ beta-rays and ${ }^{137 \mathrm{~m}} \mathrm{Ba}$ internal conversion electrons than for ${ }^{137} \mathrm{Cs}$ gamma-rays. It is also obvious from the pulse height distribution that ${ }^{137} \mathrm{Cs}$ beta-ray detection signals are distinguishable from electric noises. From these results, it is possible to count beta-rays with the photodiode through a pulse height discriminator.

\subsection{Detection efficiency for ${ }^{137}$ Cs beta-rays and gamma-rays and ${ }^{137 m} \mathrm{Ba}$ internal conversion electrons}

In the experiment with the ${ }^{137} \mathrm{Cs}$ standard source, the photodiode counted beta-rays and internal conversion electrons in proportion to the beam fluence from the source. The ratio of detection counts of internal conversion electrons to the total counts was about $10 \%$, which approximately agreed with the emission efficiency of conversion electrons in the decay of ${ }^{137} \mathrm{Cs}$.

Table 1 summarizes detection efficiency of the T-shaped tube with built-in Si PIN photodiodes for ${ }^{137} \mathrm{Cs}$ beta-rays and gamma-rays and ${ }^{137 \mathrm{~m}} \mathrm{Ba}$ internal conversion electrons. The detection efficiency for beta-rays and internal conversion electrons is defined as the ratio of the number of detection pulses to that of the particles incident upon the photodiode. The number of detection pulses of internal conversion electrons was extracted from the pulse height distribution as shown in Figure 3 (marked with slashes). The pulse height discrimination level was set at channel 150 for the case in Figure 3. The decline in the detection efficiency for ${ }^{137} \mathrm{Cs}$ beta-rays and ${ }^{137 \mathrm{~m}} \mathrm{Ba}$ internal conversion electrons is considered to be due to protection resin of the photodiode.

In the measurement of the distribution of the radioactivity in sample soil contaminated with radioactive cesium, an example of a set of measured count rate data for the photodiode pairs $(A \sim D)$ is listed in Table 2. The count rate for beta-rays was measured with the photodiode facing on the window and the other photodiode of the pair measured the count rate for gamma-rays. Naturally, the photodiodes (A, B, C) facing to the radioactive soil indicated higher count rates
Table 1. Detection efficiency of T-shaped tube for radiations from ${ }^{137} \mathrm{Cs}$

\begin{tabular}{cc}
\hline Radiation & Detection efficiency \\
\hline${ }^{137}$ Cs beta-rays & $3.0 \times 10^{-1}$ \\
Conversion electrons & $3.9 \times 10^{-1}$ \\
\hline${ }^{137}$ Cs gamma-rays & $2.0 \times 10^{-4}$ \\
\hline
\end{tabular}

Table 2. Example of measured count rate of photodiodes for sample soil.

\begin{tabular}{cccc}
\hline \multirow{2}{*}{ Photodiodes } & \multirow{2}{*}{ Depth } & \multicolumn{2}{c}{ Count rate $(\mathrm{cpm})$} \\
\cline { 3 - 4 } & & beta-rays & gamma-rays \\
\hline A & surface & 12.4 & 2.4 \\
B & surface & 11.3 & 2.3 \\
C & $10 \mathrm{~mm}$ & 14.0 & 3.2 \\
D & $30 \mathrm{~mm}$ & 0.2 & 1.0 \\
\hline
\end{tabular}

than the photodiode $\mathrm{D}$ in the lower soil layer. The beta-ray count rate for soil in Osaka was $0.14 \mathrm{cpm}$. This is the background value and originated from ${ }^{40} \mathrm{~K}$ contained in soil. In Table 2, the count rate for gamma-rays is relatively large as compared with the ratio of gamma-ray detection efficiency to beta-ray detection efficiency in Table 1 . This is because the photodiodes detected gamma-rays from a larger region compared with the case of the beta-ray detection.

It was confirmed from the sample soil experiment that the distribution of radioactive cesium in soil could be measured with the Si PIN photodiodes, which were put into the ground. Furthermore, more accurate value of radioactivity in soil is given through the comparison to reference data on standard sample soil containing the given amount of radioactivity.

\subsection{Feature comparison of photodiode and other dosimeters for radioactivity monitoring}

In addition to the Si PIN photodiodes, IPs and RPL glass dosimeters have been tested for the measurement of depth-directional distribution of radioactive cesium in soil.

The IPs and RPL glass dosimeters sensitively detect beta-rays from radioactive cesium. The IPs are easy to handle and dose data in many IP strips can be read out with a commercially available reader at one time. The RPL glass dosimeters have stable radiation sensitivity, low fading effect and reliable repeatability in radiation dose measurement, which is useful for the reduction of statistical error.

Compared with these dosimeter-type detectors, an advantage of the present beta-ray detectors with photodiodes is to be able to obtain information on radioactivity distribution in situ and in real time. This feature is useful for the evaluation of prompt change in radioactivity condition.

It should be noted that the range of a beta-ray in soil is affected by its composition, precipitation and others. Moisture could affect not only the range of a beta-ray but life span of the detector. In the examination of life span of the photodiodes, no degradation of the detection 
of beta-rays was observed for one week continuous measurement. It is, however, necessary to test longer-term stability and durability of the photodiodes under harder condition such as in the rain.

\section{Conclusion}

In this study, a detector for radioactive cesium in soil was developed by means of beta-ray counting using $\mathrm{Si}$ PIN photodiodes. Basic characteristics of the photodiode for beta-rays and gamma-rays were examined with a ${ }^{137} \mathrm{Cs}$ standard radiation source. It was confirmed that the photodiode had sufficiently higher detection efficiency for beta-rays than for gamma-rays and also detection signals of beta-rays from radioactive cesium could be successfully discriminated from gamma-ray background and electric noises. The detection efficiency of the photodiode for ${ }^{137} \mathrm{Cs}$ beta-rays and gamma-rays and ${ }^{137 \mathrm{~m}} \mathrm{Ba}$ internal conversion electrons was estimated from experiments with a ${ }^{137} \mathrm{Cs}$ beta-ray beam and under different fluxes of gamma-rays. It was found that the depth-directional distribution of radioactive cesium in soil could be measured with the tube with built-in Si PIN photodiode pairs.

\section{References}

[1] Q. He and D. E. Walling, The distribution of Fallout $137 \mathrm{Cs}$ and $210 \mathrm{~Pb}$ in undisturbed and cultivated soils, Appl. Radiat. Isot. 48 (1997), pp. 677-690.

[2] Y. A. Ivanov, N. Lewyckyj, S. E. Levchuk, B. S. Prister, S. K. Firsakova, N. P. Arkhipov, A. N. Arkhipov, S. V. Kruglov, R. M. Alexakhin, J. Sandalls and S. Askbrant, Migration of 137Cs and 90Sr from Chernobyl fallout in Ukrainian, Belarussian and Russian soils, J. Environ. Radioactivity 35 (1997), pp. 1-21.

[3] H. Kato, Y. Onda and M. Teramage, Depth distribution of 137Cs, 134Cs, and 131I in soil profile after Fukushima Dai-ichi Nuclear Power Plant Accident, J. Environ. Radioactivity 111 (2012), pp. 59-64.

[4] N. Zushi, Y. Ikeda, F. Sato, Y. Kato and T. Iida, A simple method with imaging plates for examination of soil contaminated with radioactive caesium, $J$. Nucl. Sci. Technol. 49 (2012), pp. 663-666.

[5] N. Zushi, Y. Ikeda, F. Sato, Y. Kato, K. Shimizu, N. Sato, T. Yamamoto and T. Iida, Measurement of beta-radioactivity distribution in soil by use of fiber-type radiophotoluminescence glass dosimeter, Proc. 7th Int. Workshop on Ioniz. Radiat. Monit., Oarai, Japan, Dec. 3-4, 2011, (2011), pp. 405-407. 